\title{
Treatment for Glioblastoma Multiforme Using Solid Lipid Nanoparticles with Grafted Melanotransferrin Antibody
}

\author{
Yung-Chih Kuo, In-Wei Chao \\ National Chung Cheng University \\ Chia-Yi, Taiwan 62102, Republic of China \\ chmyck@ccu.edu.tw; david821019@gmail.com
}

\begin{abstract}
Solid lipid nanoparticles (SLNs) comprising complex internal lipids were conjugated with melanotransferrin antibody (MA) to carry anticancer etoposide across the blood-brain barrier (BBB) for managing glioblastoma multiforme (GBM). MA was crosslinked on the surface of etoposide-loaded SLNs (ETP-SLNs) to target human brain-microvascular endothelial cells (HBMECs) and U87MG cells. The experimental evidences showed that an increase in the tripalmitin weight percentage in lipids enhanced the particle size and viability of U87MG cells, however decreased the etoposide loading efficiency, MA conjugation efficiency, and permeability coefficient for etoposide across the BBB. A high level of MA on the particle surface increased the atomic ratio of nitrogen to phosphorus and permeability coefficient for propidium iodide and etoposide across the BBB, however reduced the MA conjugation efficiency, transendothelial electrical resistance, and viability of U87MG cells. Based on immunochemical staining, we found that MA on ETPSLNs triggered the melanotransferrin-mediated transcytosis and promoted the growth-inhibitory efficacy to U87MG cells. MAconjugated ETP-SLNs can be a promising colloidal delivery system for malignant GBM pharmacotherapy.
\end{abstract}

Keywords: Melanotransferrin; Blood-brain barrier; Glioblastoma multiforme; Solid lipid nanoparticle; Etoposide

\section{Introduction}

Malignant glioma is a general reason of cancer cause to death, constitutes $78 \%$ of malignant brain tumors and $40 \%$ of primary brain tumors [1]. In the four categories of glioma, glioblastoma multiforme (GBM) is the highest grade malignancy that median survival of patients for only 12-15 months after therapy [2]. Therefore, GBM medication is important in clinical practice. Several challenges to treat GBM come from the following reasons: aggressive phenotype proliferation and infiltration in the cerebrum, the difficulty in early stage detection, strong resistance to traditional tumor management, and high rate of recurrence [3]. In fact, the modality of current chemotherapy for GBM does not cure cancer and usually induce serious adverse responses [4]. In order to improve the treatment of GBM, the development of adjuvant chemotherapeutics becomes one of unavoidable biomedical issues [5].

Etoposide (VM-26; VP-16) is a glycosidic derivative from the extract of Mayapple Podophyllum peltatum which belongs to a herbaceous perennial plant in the Berberidaceae family and had been restrained from medicinal utilization by its toxicity for a long time [6]. The application of etoposide to antitumor medication was approved by U.S. Food and Drug Administration through various clinical trials in 1983 [7]. Etoposide binds human type IIA topoisomerase, an enzyme involved in the cleavage of phosphodiester linkage of deoxyribonucleic acid (DNA) through nucleophilic interaction, breaks the DNA strands, and induces apoptosis [8]. However, the blood-brain barrier (BBB) might decrease the drug efficiency for 28 children with therapy of brain tumors in a phase two study on the chemotherapy of etoposide [9].

Melanotransferrin was first noticed in melanoma cells with an evident localization on the membrane via glycosylphosphatidylinositol anchorage $[10,11]$. The membrane melanotransferrin was found to participate in iron transport and metabolism, which was irrelevant to transferrin and transferrin receptor [12, 13]. In addition to iron uptake, melanotransferrin played a novel role in the transcytosis across the BBB [14].

Lipid nanoparticles have been widely applied to the therapy for glioblastoma. For example, lipid nanoparticles load edelfosine comprising Compritol 888 ATO or Precirol ATO 5 were used to pass through the BBB and inhibit the growth of C6 glioma in mice [15].

Bevacizumab, an antivascular endothelial growth factor antibody, solid lipid nanoparticles (SLNs) could increase the concentration of bevacizumab to across human umbilical vein endothelial cells for the therapy of glioblastoma [16]. 
The aim of this study was to develop etoposide-loaded SLNs (ETP-SLNs) with surface melanotransferrin antibody for GBM treatment. Since melanotransferrin is a membrane-bound protein on endothelial and tumor cells, it may provide an useful pathway in transfer anticancer drug to treat the brain cancer. We use the MA-ETP-SLNs in delivering etoposide across the BBB to inhibit the GBM growth.

\section{Materials and Methods}

\subsection{Preparation of MA-ETP-SLNs}

Cacao butter (aka cocoa butter; CB; OCG Cacao, Whitinsville, MA), tripalmitin (1,2,3-propanetriyl trihexadecanoate; palmitic triglyceride; TP; Fluka, Buchs, Switzerland), cardiolipin (1',3'-bis[1,2-dimyristoyl-sn-glycero-3-phospho]-snglycerol; CL; Avanti Polar Lipids, Alabaster, AL), etoposide (Sigma-Aldrich, St. Louis, MO), and 1,2-distearoyl-sn-glycero3-phosphoethanolamine-N-[carboxy(polyethyleneglycol)-2000] (DSPE-PEG(2000)-COOH; Avanti Polar Lipids) were stirred in methanol (J. T. Baker, Phillipsburg, NJ) at $500 \mathrm{rpm}$ and $25^{\circ} \mathrm{C}$ for $30 \mathrm{~min}$. The solute weight in methanol solution was fixed at 4\% (w/v). Cardiolipin and DSPE-PEG(2000)-COOH were controlled at $10 \%(\mathrm{w} / \mathrm{w})$ and $2.5 \%(\mathrm{w} / \mathrm{w})$ in the solutes of methanol solution. The weight percentage of etoposide in the solutes of methanol solution was $2.5 \%, 5 \%$, or $7.5 \%$. The weight percentage of tripalmitin in the solutes of methanol solution was $0 \%, 20 \%, 40 \%, 60 \%$, or $80 \%$. In addition, the surfactant solution contained 0.4\% (w/v) cholesteryl hemisuccinate (Sigma-Aldrich), 0.4\% (w/v) L-A-phospatidylcholine type II-S (Sigma-Aldrich), 0.2\% (w/v) taurocholate (Sigma-Aldrich), and 2\% (w/v) n-butanol (Riedel-de Haen, Seelze, Germany) in ultrapure water (Barnstead, Dubuque, IA). The methanol solution of $10 \mathrm{~mL}$ was emulsified with the surfactant solution of $50 \mathrm{~mL}$ using a homogenizer (PT 2100, Kinematica AG, Lucerne, Switzerland) at $25000 \mathrm{rpm}$ and $25^{\circ} \mathrm{C}$ for 10 min. Ultrapure water of $140 \mathrm{~mL}$ was added to the emulsified fluid at $400 \mathrm{rpm}$ for $1 \mathrm{~h}$. Thus, the diffusion of methanol in the aqueous phase diluted the content of residual methanol in the microemulsion. Fluorescein isothiocyanate (Sigma-Aldrich) of $50 \mathrm{ppm}$ was mixed in methanol solution for fabricating fluorescent SLNs. The suspension of ETP-SLNs was added to a cellulosic membrane bag (Spectrum Laboratories, Rancho Dominguez, CA) of $50 \mathrm{kDa}$ in a flask of $500 \mathrm{~mL}$, dialyzed against ultrapure water of $200 \mathrm{~mL}$ at $150 \mathrm{rpm}$ for $1 \mathrm{~h}$, and strained through a filter with pores of $1 \mu \mathrm{m}$. The strained suspension was centrifuged using a centrifuge (AVANTij-25, Beckman Coulter, Palo Alto, CA) at $159000 \times g$ and $4^{\circ} \mathrm{C}$ for $10 \mathrm{~min}$. The bottom solids were suspended in ultrapure water with $1 \%(\mathrm{w} / \mathrm{v}) \mathrm{D}$-mannitol (Sigma-Aldrich), frozen in a freezer (Panasonic, Gunma, Japan) at $-80^{\circ} \mathrm{C}$ for $30 \mathrm{~min}$, dried using a lyophilizer (Eyela, Tokyo, Japan) at 266.64-533.29 $\mathrm{Pa}$ and $-80^{\circ} \mathrm{C}$ for $24 \mathrm{~h}$, and preserved in a refrigerator at $4^{\circ} \mathrm{C}$. After centrifugation, the quantity of free etoposide was evaluated using a high performance liquid chromatograph (HPLC; Jasco, Tokyo, Japan) with a reverse phase BDS Hypersil C-18 column (Thermo Hypersil-Keystone, Bellefonte, PA). In the column, an aqueous acetonitrile (BDH, Poole, England) solution was impelled using two high-pressure pumps (PU-2080 Plus, Jasco). The mobile phase of acetonitrile gradient from 5\% (v/v) to 45\% (v/v) flowed at a rate of $1 \mathrm{~mL} / \mathrm{min}$ for $20 \mathrm{~min} .22$ The effluent from HPLC was analyzed using an ultraviolet (UV)-visible detector (UV-2075 Plus, Jasco) at $284 \mathrm{~nm}$. The etoposide loading efficiency, $E_{\mathrm{ETP}}$, was defined as [(weight of etoposide used - weight of etoposide in fluid)/(weight of etoposide used)] $\times 100 \%$. The suspension of ETP-SLNs was mixed with $0.05 \%(w / v) ~ N-$ hydroxysuccinimide (NHS; Acros, Morris, NJ) and 0.1\% (w/v) 1-ethyl-3-(3-dimethylaminopropyl) carbodiimide (EDC; Sigma-Aldrich) at $80 \mathrm{rpm}$ and $25^{\circ} \mathrm{C}$ for $1.5 \mathrm{~h}$, conjugated with MA (monoclonal immunoglobulin (Ig) G-2a, Santa Cruz Biotechnology, Dallas, TA) at $200 \mathrm{rpm}$ and $25^{\circ} \mathrm{C}$ for $4 \mathrm{~h}$, and centrifuged at $148000 \times g$ and $4^{\circ} \mathrm{C}$ for $10 \mathrm{~min}$. NHS and EDC were used to activate carboxyl groups of DSPE-PEG(2000)-COOH on ETP-SLNs. The chemical reaction in the conjugation of MA on ETP-SLNs enabled the formation of peptide bonds between carboxyl groups of DSPE-PEG(2000)-COOH and amino groups of MA. The quantity of MA in supernatant was determined with a QuantiPro bicinchoninic acid assay kit (protein concentration of $0.5-30 \mu \mathrm{g} / \mathrm{mL}$, Sigma-Aldrich) using an enzyme-linked immunosorbent assay (ELISA) spectrophotometer (Bio-tek, Winooski, VT) at $562 \mathrm{~nm}$. The MA conjugation efficiency on MA-ETP-SLNs, $E_{\mathrm{MA}}$, was defined as [(weight of MA used - weight of MA in supernatant)/(weight of MA used $)] \times 100 \%$.

\subsection{Particle size and zeta potential of MA-ETP-SLNs}

A zetasizer 3000 HSA with a photon correlation spectroscope and a laser Doppler velocimeter (Malvern, Worcestershire, UK) was used to evaluate the cumulant Z-average diameter of MA-ETP-SLNs, D, and zeta potential of MA- 
ETP-SLNs, $\zeta$, at $25^{\circ} \mathrm{C}$. The suspension of MA-ETP-SLNs was controlled at $2 \mathrm{mg} / \mathrm{mL}$ in a buffer of $0.1 \mathrm{M}$ tris hydroxymethyl aminomethane (tris; Riedel-de Haen) at pH 7.4.

\subsection{XPS spectra of MA-ETP-SLNs}

The atomic distribution in the surface layer of MA-ETP-SLNs was analyzed using an X-ray photoelectron spectroscope (XPS, Kratos, Kanagawa, Japan). The suspension of MA-ETP-SLNs in tris buffer of $10 \mu \mathrm{L}$ and $2 \mathrm{mg} / \mathrm{mL}$ was dropped on a cover slide of $5 \times 5 \mathrm{~mm}$ and dried at a vacuum grade of $2 \times 10-7 \mathrm{~Pa}$ and $300 \mathrm{~W}$ in a light bean area of $300 \times$ $700 \mu \mathrm{m}$ for $15 \mathrm{~min}$.

\subsection{Statistics}

All of the data were presented as mean \pm standard deviation. Statistics of data between groups were analyzed via a one-way analysis of variance (ANOVA) for independent samples and Tukey' s posthoc test was used to compare the group pairs if a significant difference was observed. In the statistical analysis, $p<0.05$ was considered to be a significant difference.

\section{Results and Discussion}

\subsection{Average particle diameter and zeta potential of MA-ETP-SLNs}

When the tripalmitin weight percentage changed, the zeta potential of MA-ETP-SLNs fluctuated between $22 \mathrm{mV}$ and $36 \mathrm{~m}$. This show that the different composition of tripalmitin could change the electrophoretic mobility of MA-ETP-SLNs. In addition, when the lipid composition (tripalmitin content) varied, the charged species on the surface, such as surfactants and phospholipids, could lead to a different size of MA-ETP-SLNs.

\subsection{MA conjugation efficiency on MA-ETP-SLNs}

An increase in the MA concentration decreased the MA conjugation efficiency. The reason was a high MA concentration enhanced the competition between free MA molecules to grafted on the carboxyl groups of DSPE-PEG(2000)$\mathrm{COOH}$ on the surface of MA-ETP-SLNs. An increase in the tripalmitin weight percentage decreased the MA conjugation efficiency. This could be explained by the fact that a high tripalmitin weight percentage enlarged the particle size and decreased the specific area for the association between MA and MA-ETP-SLNs.

\subsection{Surface atoms on MA-ETP-SLNs}

The XPS spectra of MA-ETP-SLNs show the signals of phosphorus, sulfur, carbon, nitrogen, and oxygen at $132 \mathrm{eV}$, $169 \mathrm{eV}, 283 \mathrm{eV}, 399 \mathrm{eV}$, and $531 \mathrm{eV}$, respectively. The phosphorus atoms came from CL, DSPE-PEG(2000)-COOH, and L-A-phospatidylcholine type II-S. The nitrogen atoms were from MA, taurocholate, and L-A-phospatidylcholine type II-S. An increase in the MA concentration enhanced the atomic ratio of nitrogen to phosphorus proved that the quantity of MA on the surface of MA-ETP-SLNs increased.

\subsection{TEER and permeability coefficient for PI across the BBB}

When the concentration of MA increase would decreased the TEER and enhanced the BBB permeability coefficient for PI. This suggested that the structure of tight junction in the BBB might be destroyed by MA-ETP-SLNs partially. However, the deterioration in the BBB integrity was very minor because the TEER and BBB permeability coefficient for PI were higher than $180 \Omega \times \mathrm{cm} 2$ and lower than $6.5 \mathrm{~cm} / \mathrm{s}$. This show that the MA-ETP-SLNs did not strongly damage the tight junction. Therefore, MA-ETP-SLNs could be appropriate for the transport of etoposide across the BBB.

\subsection{Viability of HBMECs, HAs, and U87MG cells treated with MA-ETP-SLNs}

The four formulations were all comparable to the control group. Firstly, etoposide is a cytotoxic anticancer agent. However, the viability of HBMECs and HAs was higher than $89 \%$ and $91 \%$, suggesting that the toxic level was acceptable. Secondly, SLNs were placebo without etoposide, low cytotoxicity was at a similar toxic level to the control groups. Thirdly, an encapsulation of etoposide in SLNs decreased the drug toxicity and enhanced the survival of HBMECs and HAs. Fourthly, 
a conjugation of MA promoted the interaction between the carriers and cells and enhanced the targeting efficiency. An encapsulation of etoposide in MA-ETP-SLNs could strongly decrease the cytotoxicity to HBMECs and HAs. Therefore, MA-ETP-SLNs might be used for medical treatment.

\section{Conclusion}

The particle core of MA-ETP-SLNs were made by various lipid ingredients in particle core and grafting MA on particle surface to transport etoposide, permeate the BBB, and inhibit the growth of GBM. The viability of HBMECs was higher than $89 \%$ and that of HAs was higher than $91 \%$ means the toxicity of MA-ETP-SLNs to the BBB cells was acceptable. Surface conjugation of MA enhanced the targeting and transcytotic transport of MA-ETP-SLNs to HBMECs and U87MG cells. Therefore, MA-ETPSLNs can be effective nanocarriers in delivering etoposide across the BBB and inhibit the growth of GBM for chemotherapeutic application.

\section{Acknowledgements}

This work is supported by the Ministry of Science and Technology of the Republic of China.

\section{References}

[1] T. Fujimaki, "Central nervous system germ cell tumors: classification, clinical features, and treatment with a historical overview," J. Child Neurol, vol. 24, pp. 1439-1445, 2009.

[2] L. Cheng, S. Bao, and J. N. Rich, "Potential therapeutic implications of cancer stem cells in glioblastoma." Biochem. Pharmacol., vol. 80, pp. 654-665, 2010.

[3] R. J. Packer, T. MacDonald, and G. Vezina, "Central nervous system tumors," Hematol. Oncol. Clin. North Am, vol. 24, pp. 87-108, 2010.

[4] J. Belda-Iniesta, J. de Castro Carpeno, E. Casado Saenz, P. Cejas Guerrero, R. Perona, and M. Gonzalez Baron, "Molecular biology of malignant gliomas," Clin. Transl. Oncol., vol. 8, pp. 635-641, 2006.

[5] J. P. Brown, R. M. Hewick, I. Hellstrom, K. E. Hellstrom, R. F. Doolittle, and W. J. Dreyer, "Human melanomaassociated antigen p97 is structurally and functionally related to transferring." Nature, vol. 296, pp. 171-173, 1982.

[6] M. Gordaliza, P. A. Garcia, J. M. del Corral, M. A. Castro, and M. A. Gomez-Zurita, "Podophyllotoxin: distribution, sources, applications and new cytotoxic derivatives." Toxicon., vol. 44, pp. 441-459, 2004.

[7] K. R. Hande, "Etoposide: four decades of development of a topoisomerase II inhibitor," Eur. J. Cancer., vol. 34, pp. 1514-1521, 1998.

[8] Y. Pommier, E. Leo, H. Zhang, and C. Marchand, "DNA topoisomerases and their poisoning by anticancer and antibacterial drugs," Chem. Biol., vol. 17, pp. 421-433, 2010.

[9] M. N. Needle, P. T. Molloy, J. R. Geyer, A. Herman-Liu, B. J. Belasco, J. W. Goldwein, L. Sutton, and P. C. Phillips. "Phase II study of daily oral etoposide in children with recurrent brain tumors and other solid tumors," Med. Pediatr. Oncol., vol. 29, pp. 28-32, 1997.

[10] T. M. Rose, G. D. Plowman, and D. B. Teplow, "Dreyer WJ, Hellstrom KE, Brown JP. Primary structure of the human melanomaassociated antigen p97 (melanotransferrin) deduced from the mRNA sequence," in Proc. Natl. Acad. Sci. USA, vol. 83, pp. 1261-1265, 1986.

[11] M. L. Kennard, D. R. Richardson, R. Gabathuler, P. Ponka, and W. A. Jefferies, "A novel iron uptake mechanism mediated by GPI anchored human p97," EMBO J., vol. 17, pp. 4178-4186, 1995.

[12] E. Sekyere and D. R. Richardson, "The membrane-bound transferrin homologue melanotransferrin: roles other than iron transport," FEBS Lett., vol. 483, pp. 11-16, 2000.

[13] Y. S. Rahmanto, L. L. Dunn, and D. R. Richardson. "The melanoma tumor antigen, melanotransferrin (p97): a 25-year hallmark - from iron metabolism to tumorigenesis," Oncogene., vol. 26, pp. 6113-6124, 2007.

[14] Y. S. Rahmanto, S. Bal, K. H. Loh, Y. Yu, and D. R. Richardson. "Melanotransferrin: search for a function," Biochim. Biophys. Acta., vol. 1820, pp. 237-243, 2012.

[15] A. E. de Mendoza, V. Preat, F. Mollinedo, and M. J. Blanco-Prieto, "In vitro and in vivo efficacy of edelfosine-loaded lipid nanoparticles against glioma," J. Control. Release, vol. 156, pp. 421-426, 2011. 
[16] L. Battaglia, M. Gallarate, E. Peira, D. Chirio, I. Solazzi, S. M. A. Giordano, C. L. Gigliotti, C. Riganti, and C. Dianzani, "Bevacizumab loaded solid lipid nanoparticles prepared by the coacervation technique: preliminary in vitro studies," Nanotechnology, vol. 26, pp. 255102, 2015. 